\title{
An Overview of Amniotic Fluid Embolism: Past, Present and Future Directions
}

\author{
Taihei Tsunemi, Hidekazu Oi, Toshiyuki Sado, Katsuhiko Naruse, Taketoshi Noguchi and \\ Hiroshi Kobayashi*
}

Department of Obstetrics and Gynecology, Nara Medical University, Nara, Japan

\begin{abstract}
Objective: A recent report has highlighted that amniotic fluid embolism (AFE) is the first among maternal mortality in Japan. The clinical presentation is not the same with respect to symptoms, timing and subsequent course.

Methods: This article reviews the English language literature for pathophysiology on AFE based on the clinical and animal studies.

Results: First, AFE syndrome may be divided into three subgroups designated the classical subtype, the anaphylactoid subtype and the DIC subtype, each having a distinct pattern of clinical symptoms and disease severity. Second, AFEassociated reactions can be classified as an anaphylactoid reaction or complement activation to fetal antigens or an idiosyncratic reaction. Host idiosyncrasy may be a major cause of hypersensitivity reaction. Third, the AFE reaction may be caused by a combination of immunologic and vasospastic factors. Finally, the development of effective markers for diagnosing entry of amniotic fluid into the maternal circulation would have an impact on early diagnosis and AFE-related mortality.

Conclusion: This review summarizes new insights into the pathophysiology of AFE, with a focus on the potential direction of future research.
\end{abstract}

Keyword: Amniotic fluid embolism, Anaphylactoid, Hypersensitivity, Meconium, Vasospasm.

\section{INTRODUCTION}

Amniotic fluid embolism (AFE) is a significant worldwide health problem. This disorder was first described by Meyer in 1926 [1], gaining widespread recognition by Steiner and Lushbaugh in 1941 [2]. This syndrome is diagnosed on the basis of its characteristic clinical features of sudden onset of cardiopulmonary arrest, acute hypoxia, acute hypotension, hemodynamic instability, or consumption coagulopathy, and the absence of other illness that could explain the signs and symptoms [3]. The most frequent mode of clinical presentation of AFE is acute respiratory failure and cardiovascular collapse. Despite its rare occurrence $(\sim 5$ per 100,000 birth), AFE is a major obstetric catastrophic illness in the peripartum or postpartum period, with a high mortality rate $(20-50 \%)$, and is the major cause of maternal mortality [4-6]. The underlying mechanism for AFE is still poorly understood, but it is clear that sufficient amniotic fluid, meconium and fetal debris have to enter the maternal circulation.

Recent evidence suggests that the occurrence of AFE is not a consequence of the "simple" mechanical respiratory obstruction, but a humoral effect causing anaphylactoid reactions [4] or complement activation [7]. In this review we will summarize and update the current knowledge with respect to

*Address correspondence to this author at the Department of Obstetrics and Gynecology, Nara Medical University, 840 Shijo-cho, Kashihara, 634-8522, Japan; Tel: +81-744-29-8877; Fax: +81-744-23-6557;

E-mail: hirokoba@naramed-u.ac.jp this intriguing and complex new hallmark of AFE, paying special attention to the subtypes in AFE.

\section{MATERIALS AND METHODS}

The present article reviews the literatures for clinical, biological, pathogenetic and pathophysiological studies on AFE. For studies that reported data on the obstetric disorders, data pertaining to AFE and anaphylaxis were included. A computerized literature search was performed to identify relevant studies reported in the English language. MEDLINE updates were conducted monthly, and all abstracts were reviewed by two investigators to identify papers for full-text review. We initially searched PubMed MEDLINE electronic databases (http://www.ncbi.nlm.nih.gov/sites/entrez) for a 30-year period (1980-2010), combining the keywords "pathogenesis" " "pathophysiology" " anaphylaxis" " anaphylactoid" "mediator" "tryptase" "sialyl Tn" "zinccoproporphyrin" "amniotic fluid" "meconium" "vasospasm" "vasoconstriction" or "model" with "AFE". Additionally, references in each article were searched to identify potentially missed studies. Target publications are mainly reports on human AFE and animal models, as well as studies in gene and protein expression systems. As the main interest is AFE obtained from human samples, we have not yet included animal preeclampsia model alone. However, we included the animal studied performed to support clinical data. A priori, case reports, and abstracts were not included, since abstracts do not undergo a stringent peer review process. We 
finally discuss the conventional marker for AFE diagnosis and novel biomarker candidates.

Initially, 202 potentially relevant articles were identified by screening electronic databases. 59 peer-reviewed journal articles were additionally identified from referrences in each article. 16 articles were excluded because of duplicated publication. In the end, 34 publications with full text papers displaying a focus on AFE were retrieved and reviewed. The data drawn from the previously published report [8] was recalculated in this review.

\section{The Major Causes of Maternal Death}

The main causes of maternal mortality worldwide are eclampsia/preeclampsia (pregnancy-induced hypertension, PIH) (28\%), indirect causes (26\%; AIDS, anemia, malaria, and cardiac disease), hemorrhage (20\%), infection (18\%), unsafe abortion (4\%) and other direct causes [9]. On the other hand, obstetric hemorrhage, thromboembolism, preeclampsia/PIH, primary infection, cerebral bleeding and AFE remain the leading causes of maternal death in developed countries $[10,11]$. In Japan, maternal mortality was relatively high over 20 years ago. The reported maternal mortality rates in 1990 for Japan, the United States, and United Kingdom were 8.6, 8.2, and 7.6 per 100,000 live births, respectively. Hemorrhage (38\%), intracranial hemorrhage (14\%), PIH (9\%), pulmonary thromboembolism (9\%), AFE (4\%), and other direct causes are the most frequently reported causes of maternal deaths $[12,13]$. Recently, maternal mortality rate decreased dramatically in Japan. It reached 3.2 per 100,000 , one of the lowest in the world. Furthermore, perinatal and infant mortality rates were the lowest worldwide [13]. Interestingly, more recent investigation in Japan showed that AFE, disseminated intravascular coagulation (DIC) related to PIH, pulmonary thromboembolism and injury to the birth canal were the major causes of maternal death [14]. AFE is the first among maternal mortality. The current estimated incidence for AFE is 1 in 50,000 deliveries $[15,16]$. AFE diagnosed by autopsy in Japan was often clinically diagnosed as atonic bleeding or DIC of unknown cause [14].

\section{Definition of AFE}

Better definition of the clinical signs and symptoms would have potential importance in AFE. However, different entry/diagnostic criteria have been recommended by different expert groups. The following is entry criteria for suspected AFE consisting of the presence of the following four factors in the United States [4] and in the United Kingdom [17]: 1) acute hypotension or cardiac arrest; 2) acute hypoxia; 3 ) coagulopathy or severe clinical hemorrhage in the absence of other explanations; and 4) all of these occurring during labor, cesarean delivery, or dilation and evacuation or within 30 minutes postpartum with no other explanation for the clinical findings.

On the other hand, in Japan, the diagnosis of AFE is based on a combination of the clinical features listed in the Japan Consensus Criteria for the Diagnosis of AFE established in 2002 (Table 1). AFE has been classified as fatal (pathologically confirmed; fatalities) or non-fatal (survivors). Definite diagnosis can be confirmed by identification of lanugo, fetal hair and fetal squamous cells (squames) in the histological studies at autopsy or in blood aspirated from the right ventricle [18]. The combination of clinical symptoms with detection of these cells is required. Inclusion criteria were as follows: 1) at least one of the following symptoms; cardiac arrest (acute hypoxia and hypotension), respiratory arrest (dyspnea), or consumptive coagulopathy (severe obstetric hemorrhage), 2) onset of all of the signs and symptoms developed during pregnancy, labor, cesarean section, or within $12 \mathrm{~h}$ postpartum, and 3) the absence of other illness that could explain the signs and symptoms. The study subjects were drawn from the Japan AFE Registration Center in Hamamatsu University School of Medicine, Shizuoka, which is closely linked to the AFE Association of Japan, Nara Medical University. Study population and sample collection have been described elsewhere [8].

\section{Table 1. The Japan Consensus Criteria for the Diagnosis of AFE}

A. The fatal AFE (fatalities)*:

The diagnosis is made on the basis of clinical presentation after excluding differential diagnosis and at autopsy in the event of death of the parturient. The diagnosis was confirmed by histochemical studies in the established individuals.

B. The non-fatal AFE (survivors):

The survivors have the hallmark clinical manifestations of AFE.

1. Signs and symptoms

Cardiac arrest (acute hypoxia and hypotension)

Respiratory arrest (dyspnea)

Consumptive coagulopathy (disseminated intravascular coagulation)

2. Onset of all of the signs and symptoms developed during pregnancy, labor, cesarean section or within $12 \mathrm{~h}$ postpartum

3. The absence of other illness that could explain the signs and symptoms described above

*,Definite diagnosis can be confirmed by identification of lanugo, fetal hair and fetal squamous cells (squames) in the histological studies at autopsy or in blood aspirated from the right ventricle (16).

Analysis of the Japan registry revealed that AFE occurred during labor, before delivery and within 30 min postpartum in $17 \%$ of cases. The remaining AFE cases (83\%) had symptoms more than 30 min after delivery: the average time from delivery to symptom onset was $3.3 \mathrm{~h}$ and the standard deviation was $4.3 \mathrm{~h}$ [8]. These patients were related to DIC, eventually causing bleeding manifestations [8]. AFE is considered as a cause of a coagulopathy. All AFE patients who had clinical symptoms within $12 \mathrm{~h}$ postpartum period were included in the Japan AFE Registration when there is no other explanation for the clinical findings. Different criteria used for diagnosing AFE would provide differing results.

\section{Impact on Clinical Symptoms and Disease Severity}

Oi et al., retrospectively analyzed the data in AFE patients registered in the Japan AFE Registration Center (19922006) [8]. Totally 135 patients were analyzed, with 65 fatal AFE and 70 non-fatal AFE. Although respiratory distress, 
cardiac dysfunction and massive bleeding were three main manifestations of the patients, the mode of death or clinical manifestations are not the same for each cause. We have proposed a model of AFE that takes into account the diverse nature of this syndrome and correlates the clinical and pathological features of the disease.

In this model, AFE was divided into three categories (subgroups), on the basis of symptoms at presentation, designated the classical subtype (respiratory arrest or distress), the anaphylactoid subtype (cardiac arrest or ventricular dysfunction) and the DIC subtype (massive bleeding): the classical subtype in 41 cases, the anaphylactoid subtype in 46 cases, the DIC subtype in 48 cases, a combination of the two categories in 52 cases, and a combination of the three categories in 52 cases (Table 2). There is overlap between these subgroups and clinical characteristics associated with AFE. Therefore, the number of each pure subgroup is low. The data drawn from the previously published report (ref. [8]) was recalculated and summarized in Table 2. The impact on mortality of each AFE subgroup has been evaluated. Mortality rate was $53.7 \%$ in the classical subtype, $39.1 \%$ in the anaphylactoid subtype and $25.0 \%$ in the DIC subtype, demonstrating that each subgroup has a distinct pattern of disease severity. The classical subgroup has a higher frequency of sudden death when compared to the DIC subgroups. Both clinical and autopsy signs of DIC were essentially absent in the case of sudden death. The prognosis of the DIC subgroup is relatively good. Early detection and adequate treatment of DIC can improve the prognosis of AFE [19].

\section{Similarities, Overlap and Differences in AFE Subgroups}

The aim of this section was to provide an overview of the pathophysiology of AFE subgroups (Table 3).

First, non-thrombotic pulmonary embolism (NTPE) is a disease entity that is commonly defined as the partial or total occlusion of the pulmonary circulation [20]. Amniotic fluid is one of the main sources of NTPE [21]. Previous theories published more than 70 years ago attributed AFE to acute obstruction of maternal pulmonary vessels. In light of recent study results, this embolization theory ("simple" mechanical obstruction) may be abandoned. However, the embolization by massive amniotic debris (epithelial squamous cells, granules of meconium-derived bile pigment, and even several lanugo hairs) might lead to sudden respiratory arrest, pulmonary hypertension, right heart failure, hypoxia and subsequent death.

Laboratory tests can only be helpful for establishing the cause of the disease. Kobayashi et al., have initially reported a significant high serum level of sialyl Tn (STN) antigen in the AFE cases $(110.8 \pm 48.1 \mathrm{U} / \mathrm{ml}$ for AFE vs. $17.3 \pm 2.6$ $\mathrm{U} / \mathrm{ml}$ for control) [22]. STN is a glycoprotein derived from human meconium $[22,23]$. The method for detecting STN in the maternal serum of patients with AFE is a direct way to demonstrate the release of meconium-derived mucin into the maternal circulation and is a simple and noninvasive method for diagnosis of AFE [22,23].

The patients who died had significantly higher concentrations of STN than the survivors $(p=0.003$, ref. [8]). As shown in Table 2, the median serum STN levels were significantly higher in patients with the classical subtype (median, 25.3 $\mathrm{U} / \mathrm{ml}$; range, 3.1-324 U/ml) compared with patients with the anaphylactoid subtype $(18.1 \mathrm{U} / \mathrm{ml} ; 3.1-1320 \mathrm{U} / \mathrm{ml}$, $\mathrm{p}=0.0571)$ and those with the DIC subtype $(16.4 \mathrm{U} / \mathrm{ml} ; 10$ $1320 \mathrm{U} / \mathrm{ml}, \mathrm{p}=0.0158)$, respectively. Microscopically, the amniotic mucins were immunohistochemically visible as STN-positive structures [23]. These findings form the "classical" pathologic findings in AFE. Deaths from this type tend to be characterized by an immediate onset (within minutes) and progression to arrest. Such immediate progression makes treatment more difficult [24].

Second, about 15 years ago, Clark et al., have proposed renaming this disorder "anaphylactoid syndrome of preg-

Table 2. The Impact on Mortality and Serum STN Levels in Each Subgroup

\begin{tabular}{|c|c|c|c|c|}
\hline Subtype & $\mathbf{n}$ & The classical subtype [C] & The anaphylactoid subtype $[\mathrm{A}]$ & The DIC subtype [D] \\
\hline$[\mathrm{A}]$ & 4 & - & 4 & - \\
\hline [D] & 10 & - & - & 10 \\
\hline$[\mathrm{C}]+[\mathrm{A}]$ & 14 & 14 & 14 & - \\
\hline$[\mathrm{A}]+[\mathrm{D}]$ & 28 & - & 28 & 28 \\
\hline Total & & 41 & 46 & 48 \\
\hline Mortality rate & & 53.7 & 39.1 & 25.0 \\
\hline $\begin{array}{c}\text { STN (U/ml) } \\
\text { Median (range) }\end{array}$ & & $25.3^{\mathrm{d}}(3.1-324)$ & $18.1^{\mathrm{e}}(3.1-1320)$ & $16.4^{\mathrm{f}}(10-1320)$ \\
\hline
\end{tabular}

The data drawn from the previously published report (ref. [8]) was recalculated. [C], the classical subtype; [A], the anaphylactoid subtype; and [D], the DIC subtype. Kruskal-Wallis test showed significant difference ( $\mathrm{p}=0.0221$ ), with Scheffé post hoc test indicating difference with respect to AFE mortality rate. The mortality rates from AFE patients in the C and D groups were 53.7 and $25.0 \%$, respectively, resulting in a statistically significant difference between the two groups ( $\mathrm{p}=0.0211$ ). No statistically significant interactions with respect to AFE mortality rate were observed between the $\mathrm{C}$ and $\mathrm{A}$ groups $(53.7 \%$ vs. $39.1 \%, \mathrm{p}=0.3701)$ as well as the $\mathrm{A}$ and $\mathrm{D}$ groups $(39.1 \%$ vs. $25.0 \%$, $\mathrm{p}=0.3611)$. In addition, differences in STN levels between groups were analyzed with Mann-Whitney U test. d vs. e, $p=0.0571$; $d$ vs. f, $p=0.0158$; and e vs. f, $p=0.6642$. The STN levels in AFE patients in the C and D groups were $25.3 \mathrm{U} / \mathrm{ml}$ and $16.4 \mathrm{U} / \mathrm{ml}$, respectively, resulting in a statistically significant difference between the two groups ( $\mathrm{p}=0.0158$ ). 
Table 3. A Broad Classification of AFE into Three Major Subtypes

\begin{tabular}{|c|c|c|c|c|c|}
\hline Subtype & Mechanisms & $\begin{array}{c}\text { Initial Signs and } \\
\text { Symptoms }\end{array}$ & Onset & $\begin{array}{c}\text { Prognosis: Fatal or } \\
\text { Non-Fatal }\end{array}$ & $\begin{array}{c}\text { Fetal anti- } \\
\text { gens (STN) } \\
\text { in maternal } \\
\text { serum }\end{array}$ \\
$\begin{array}{c}\text { Amniotic Fluid } \\
\text { Entering the } \\
\text { Maternal } \\
\text { Circulation }\end{array}$ \\
\hline \hline Classic & $\begin{array}{c}\text { mechanical } \\
\text { obstruction }\end{array}$ & $\begin{array}{c}\text { pulmonary dyspnea } \\
\text { and arrest }\end{array}$ & $\begin{array}{c}\text { immediate (within } \\
\text { minutes) onset }\end{array}$ & $\begin{array}{c}\text { Aggressive course: } \\
\text { fatal > non-fatal }\end{array}$ & elevated \\
\hline Anaphylactoid & anaphylaxis & $\begin{array}{c}\text { cardiac dysfunction } \\
\text { and arrest }\end{array}$ & $\begin{array}{c}\text { intermediate onset } \\
\text { fatal = non-fatal }\end{array}$ & slightly \\
elevated & moderate \\
\hline DIC & $\begin{array}{c}\text { coagulation and } \\
\text { protease attack }\end{array}$ & coagulopathy & $\begin{array}{c}\text { delayed (within } \\
\text { hours) onset }\end{array}$ & $\begin{array}{c}\text { Good response to therapy: } \\
\text { fatal < non-fatal }\end{array}$ & $\begin{array}{c}\text { slightly } \\
\text { elevated }\end{array}$ \\
\hline
\end{tabular}

There has been a greater understanding of the spectrum and pathophysiology of AFE. Recent evidence suggests that AFE is not a single disease. In the present review, AFE was classified as three distinct entities. The significant overlap exists between the classical subtype, the anaphylactoid subtype and the DIC subtype. Some of cases that are the anaphylactoid subtype are the classical subtype and also the DIC subtype. The classical subtype without the anaphylactoid or DIC subtype also exists. These overlaps are not complete.

nancy" [4]. AFE deaths resulting from maternal entry of amniotic fluid could be the result of a generalized or systemic anaphylactoid reaction $[4,18,19,25,26]$.

In general, food or venom-related anaphylaxis is the most dramatic clinical presentation of serious allergy that occurs after contact with an allergen and involves multiple organ systems. Approximately half of the fatal anaphylactic reactions were iatrogenic and a quarter each caused by foods or insect venom [29]. Anaphylactic reactions cause variable combinations of symptoms including the skin (acute urticaria, rash, angioedema, flushing, pruritis), the gastrointestinal system (nausea, vomiting, diarrhea, abdominal pain) and cardiopulmonary reactions (laryngeal edema, bronchospasm, hypotension, syncope/presyncope, tachycardia, arrhythmia, dyspnea, chest pain, wheezing, stridor, hypoxia, sudden death), from mild skin reactions to cardiopulmonary arrest, within the first minutes or hours of reaction [28]. Typical severe anaphylaxis in deaths related to food, venom or drugs may lead to shock, as a result of either direct effects of mediators on the heart, or profound shock resulting from peripheral vasodilatation [28]. The estimated incidence of food, venom or drug-related anaphylaxis was 5-10 in 100,000, with a mortality rate ranging from $1 \%$ to $5 \%$ [29]. In contrast, incidence of AFE is 2 per 100,000 with fatality in about $30 \%$ of cases, suggesting that the mortality rate in AFE is higher than that in food, venom or drug-related anaphylaxis. Although the pathophysiology has been believed to be immune mediated, a few cases with typical skin reactions, laryngeal edema and gastrointestinal symptoms have been reported in association with AFE [24]. Thus, a part of AFE syndrome is thought to have died from a reason other than anaphylaxis (anaphylactic or anaphylactoid reactions). The pathophysiology of AFE may not be the same for anaphylactoid, suggesting that other factors could interact to influence or strengthen the development of AFE

Finally, the remaining AFE cases (the DIC subtype) were accepted as being the result of peripartum and postpartum bleeding. Amniotic fluid may also act as a trigger of events resembling complement system activation [7] possibly through meconium-containing protease activities [30], subsequently resulting in DIC and multiple organ failure characteristics. The DIC subtype causes delayed cardiac arrest with massive bleeding rather than asphyxia from bronchospasm, which is seen more commonly with anaphylactoid cases. The DIC type reactions start with mild symptoms and progress over several hours. Early detection of DIC immediately after the onset of clinical symptoms can lead to curative treatment.

Taken together, AFE is not a single entity. AFE might be classified into three largely distinct entities, namely the classical subtype, the anaphylactoid subtype and the DIC subtypes (Table $\mathbf{3}$ ).

\section{New Insights into the Pathophysiology}

Why does AFE cause cardiopulmonary arrest? The cause of AFE-associated reactions seems to be classified by two hypothesis: an effect of amniotic fluid itself or a host idiosyncrasy ("hypersensitivity" reaction).

\section{Amniotic Fluid as a Causative Factor}

Hypersensitivity reactions may depend on the causative components including the infectious bacteria in amniotic fluid, suggesting that some patients with the clinical diagnosis of AFE have infection as a mechanism of disease [31]. Meconium-stained amniotic fluid may be more toxic than clear amniotic fluid. Meconium-stained amniotic fluid without infection also contains proteolytic enzymes and chemical mediators that can stimulate mast cell degranulation and complement activation. It has been reported that meconium is a vasoconstrictive agent [32]. Furthermore, trypsin and thrombin in meconium may also play a pivotal role in vasospasm.

\section{Host Idiosyncrasy as a Causative Factor}

On the other hand, more attention needs to be placed on the host factors. For example, clinical symptoms from stinging events involving honey bees are highly varied, ranging from mild skin reactions to fatal outcomes. Death is often caused by anaphylaxis, and the typical victim is over 40 years of age, with arteriosclerosis, heart disease, asthma, renal disease, or diabetes playing a contributing role [33]. Moreover, the dysfunctional cardiac conditions with ischemic heart disease and cardiomyopathy are major risk 
factors for fatal anaphylaxis [34]. These individuals with heart disease are considered to be more susceptible to the venom-induced anaphylactic shock, which often play an aggravating role.

Triggiani et al., indicated that the heart is a central organ of anaphylaxis [34]. The offensive antigens from amniotic fluid, meconium and fetus initially meet the heart. The protective function depends on the abundant number of immunocompetent effector cells in heart. Of interest is the mast cell, this cell is abundant in the heart [34]. Activation of mast cells induces the release of preformed mediators (histamine, tryptase, chymase, carboxypeptidase $\mathrm{A}$, and renin) and the de novo synthesis of leukotriene (LT) $\mathrm{C}_{4}$, prostaglandin (PG) $\mathrm{D}_{2}$, platelet activating factor and tumor necrosis factor (TNF)-alpha [35]. The release of mast cell-derived chemical mediators occurs within minutes, which initiate anaphylactic, anaphylactoid and vasospastic reactions. Of interest is the observation that, upon activation, cardiac mast cells release renin that activates the angiotensin system locally via cardiac renin-angiotensin-system (RAS) [34]. Angiotensin I, generated by renin, can be converted to angiotensin II by cardiacderived chymase with angiotensin-converting enzyme activity, which further induces strong vasoconstriction [34]. AFE may be attributed to an acute vasoconstriction. Therefore, hypersensitivity reactions of AFE may depend on host idiosyncrasy, who is more susceptible to the anaphylactic shock creased in patients with ischemic heart disease and dilated cardiomyopathy [34]. Histamine and tryptase content and mast cell density are also higher in these failing hearts. Host factors such as subclinical heart complications may be associated with susceptibility to severe manifestations of AFE. The available evidence supports a role for hypersensitivity: cardiac conditions such as pre-existing subclinical or asymptomatic heart disease including ischemic heart disease, cardiomyopathy, or endothelial dysfunction might have contributed to the presentation and severity of AFE.

Based on the present knowledge and hypothesis of the mechanisms, we will propose renaming AFE "hypersensitivity syndrome of pregnancy". The proposed terminology "hypersensitivity" is an umbrella term including vasospasm.

\section{DISCUSSION}

AFE is an obstetric medical emergency that presents a broad array of symptoms and signs. These clinical manifestations do not occur uniformly in all patients. AFE must be considered as a differential diagnosis for any acute-onset obstetric disorders. The diagnosis of AFE should be established by the clinical course. AFE represents a heterogeneous group that may arise via different pathogenetic pathways.

In this review, AFE might be divided into three subgroups designated the classical subtype, the anaphylactoid subtype and the DIC subtype, each having a distinct pattern of clinical symptoms and disease severity. First, AFE is initially believed to occur through the embolization by a large amount of amniotic fluid and debris of fetal origin (namely the classical subtype). Second, this syndrome has been later classified as an anaphylactic reaction; this process is different to the classical primary embolization. Finally, peripartum and postpartum acute hemorrhage supports the diagnosis of AFE in the fulminant DIC subgroup. A release of soluble mediators could explain the symptoms that are present in AFE. Therefore, amniotic fluid release into maternal circulation is the necessary precursor to AFE.

The present review allows us to speculate that AFEassociated reactions might be derived from amniotic fluid itself or host idiosyncratic reaction. There seems to be a case of typical anaphylactic reaction, or a case of more consistent with idiosyncratic reaction. Benson et al., suggested that hypersensitivity reaction has been frequently underdiagnosed, with mild symptoms being missed [36].

Despite this hypothesis, there is a lack of the basic knowledge of the specific antigen(s) in amniotic fluid or debris. The exact mechanisms of idiosyncratic reactions or factors involved in host specificity (hypersensitivity) are also still unclear so far. Upon reaction, the release of mast cellderived mediators occurs within minutes, which initiates anaphylactic reaction, anaphylactoid reaction, complement activation and vasospastic hypersensitivity reactions. These reactions influence vasoconstriction and ventricular function, suggesting that an important part of AFE is of known vasospastic etiology. The AFE reaction might be caused by a combination of immunologic and vasospastic factors. $\mathrm{Hu}-$ moral factors, such as cardiac mast cell-derived mediators, can exacerbate cellular responses and create complex pathways that lead to a variety of clinical manifestations. Hypersensitivity reactions of AFE may depend on host idiosyncrasy, who is more susceptible to the anaphylactic shock. These reactions can occur only in susceptible individuals: for example, pregnant women with pre-existing subclinical heart disease.

In conclusion, this review presents an AFE-induced clinical manifestation of hypersensitivity syndrome and the necessity of monitoring the patients clinically and by laboratory investigations. Through currently available data, both the hypersensitivity hypothesis and the vasospastic mechanism are able to be assessed.

\section{CONDENSATION}

This review summarizes new insights into the pathophysiology of AFE, with a focus on an evaluation of existing indicators and a potential direction for future research

\section{ACKNOWLEDGEMENTS}

\section{Grant Support}

Supported by Grant-in-aid for Scientific Research from the Ministry of Education, Science, and Culture of Japan to the Department of Obstetrics and Gynecology, Nara Medical University (H. Kobayashi).

\section{CONFLICT OF INTEREST}

None declared.

\section{REFERENCES}

[1] Meyer JR. Embolia pulmonar amnio-caseosa. Braz Med 1926; 2 : 301-3. 
[2] Steiner PE, Lushbaugh CC. Maternal pulmonary embolism by fluid as a cause of obstetric shock and unexpected deaths in obstetrics. JAMA 1941; 117: 1245-54.

[3] Tomasa TM, Castellà E, Homs M, et al. Amniotic fluid embolism diagnosed by fibre-optic bronchoscopy. Anaesthesia 2010; 65: 1230-1.

[4] Clark SL, Hankins GD, Dudley DA, Dildy GA, Porter TF. Amniotic fluid embolism: analysis of the national registry. Am J Obstet Gynecol 1995; 172: 1158-67.

[5] Kramer MS, Rouleau J, Baskett TF, Joseph KS. Maternal Health Study Group of the Canadian Perinatal Surveillance System. Amniotic fluid embolism and medical induction of labor: a retrospective, population-based cohort study. Lancet 2006; 368: 1444-8.

[6] Abenhaim HA, Azoulay L, Kramer MS, Leduc L. Incidence and risk factors of amniotic fluid embolisms: a population-based study on 3 million births in the United States. Am J Obstet Gynecol 2008; 199: 49.e1-49.e8

[7] Benson MD, Kobayashi H, Silver RK, Oi H, Greenberger PA, Terao T. Immunologic studies in presumed amniotic fluid embolism. Obstet Gynecol 2001; 97: 510-4.

[8] Oi H, Naruse K, Noguchi T, et al. Fatal factors of clinical manifestations and laboratory testing in patients with amniotic fluid embolism. Gynecol Obstet Invest 2010; 70: 138-44.

[9] Gil-González D, Carrasco-Portiño M, Ruiz MT. Knowledge gaps in scientific literature on maternal mortality: a systematic review. Bull World Health Organ 2006; 84: 903-9.

[10] Fässler M, Zimmermann R, QuackLötscher KC. Maternal mortality in Switzerland 1995-2004. Swiss Med Wkly 2010; 140: 25-30.

[11] Liang J, Zhu J, Dai L, Li X, Li M, Wang Y. Maternal mortality in China, 1996-2005. Int J Gynaecol Obstet 2010; 110: 93-6.

[12] Alexander S, Wildman K, Zhang W, Langer M, Vutuc C, Lindmark G. Maternal health outcomes in Europe. Eur J Obstet Gynecol Reprod Biol 2003; 111: S78-S87.

[13] Nagaya K, Fetters MD, Ishikawa M, et al. Causes of maternal mortality in Japan. JAMA 2000; 283: 2661-7.

[14] Kanayama N, Inori J, Ishibashi-Ueda $\mathrm{H}$, et al. Maternal death analysis from the Japanese autopsy registry for recent 16 years: significance of amniotic fluid embolism. J Obstet Gynaecol Res 2010; 37: 58-63.

[15] Roberts CL, Algert CS, Knight M, Morris JM. Amniotic fluid embolism in an Australian population-based cohort. BJOG 2010; 117: 1417-21.

[16] Knight M, Tuffnell D, Brocklehurst P, Spark P, Kurinczuk JJ. UK Obstetric Surveillance System. Incidence and risk factors for amniotic-fluid embolism. Obstet Gynecol 2010; 115: 910-7.

[17] Tuffnell DJ. United kingdom amniotic fluid embolism register. BJ0G 2005; 112: 1625-9.

[18] Rudra A, Chatterjee S, Sengupta S, Nandi B, Mitra J. Amniotic fluid embolism. Indian J Crit Care Med 2009; 13: 129-35.

[19] Jorens PG, Van Marck E, Snoeckx A, Parizel PM. Nonthrombotic pulmonary embolism. Eur Respir J 2009; 34: 452-74.
[20] Montagnana M, Cervellin G, Franchini M, Lippi G. Pathophysiology, clinics and diagnostics of non-thrombotic pulmonary embolism. J Thromb Thrombolysis 2011; 31: 436-44.

[21] Sinicina I, Pankratz H, Bise K, Matevossian E. Forensic aspects of post-mortem histological detection of amniotic fluid embolism. Int J Legal Med 2010; 124: 55-62

[22] Kobayashi H, Ohi H, Terao T. A simple, noninvasive, sensitive method for diagnosis of amniotic fluid embolism by monoclonal antibody TKH-2 that recognizes NeuAc alpha 2-6GalNAc. Am J Obstet Gynecol 1993; 168: 848-53.

[23] Kobayashi H, Ooi H, Hayakawa H, et al. Histological diagnosis of amniotic fluid embolism by monoclonal antibody TKH-2 that recognizes NeuAc alpha 2-6GalNAc epitope. Hum Pathol 1997; 28: 428-33.

[24] Ječmenica D, Baralić I, Alempijević D, Pavlekić S, Kiurski M, Terzić M. Amniotic Fluid Embolism-Apropos Two Consecutive Cases. J Forensic Sci 2011; 56: S247-51.

[25] Ebo DG, Bosmans JL, Couttenye MM, Stevens WJ. Haemodialysis-associated anaphylactic and anaphylactoid reactions. Allergy 2006; 61: 211-20.

[26] Mahshid N, Ahmad S, Nahid M, Afshin F. Sudden cardiac arrest during cesarean section -- a possible case of amniotic fluid embolism. Middle East J Anesthesiol 2009; 20: 315-7.

[27] Pumphrey RS, Roberts IS. Postmortem findings after fatal anaphylactic reactions. J Clin Pathol 2000; 53: 273-6.

[28] Balazic J, Rott T, Jancigaj T, Popović M, Zajfert-Slabe M, Svigelj V. Amniotic fluid embolism with involvement of the brain, lungs, adrenal glands, and heart. Int J Legal Med 2003; 117: 165-9.

[29] Hankins GD, Snyder R, Dinh T, Van Hook J, Clark S, Vandelan A Documentation of amniotic fluid embolism via lung histopathology. Fact or fiction? J Reprod Med 2002; 47: 1021-4.

[30] Petroianu GA, Altmannsberger SH, Maleck WH, Assmus HP, Friedberg C, Bergler WF. Meconium and amniotic fluid embolism: effects on coagulation in pregnant mini-pigs. Crit Care Med 1999; 27: 348-55.

[31] Romero R, Kadar N, Vaisbuch E, Hassan SS. Maternal death following cardiopulmonary collapse after delivery: amniotic fluid embolism or septic shock due to intrauterine infection? Am J Reprod Immunol 2010; 64: 113-25.

[32] Holcberg G, Huleihel M, Katz M, et al. Vasoconstrictive activity of meconium stained amniotic fluid in the human placental vasculature. Eur J Obstet Gynecol Reprod Biol 1999; 87: 147-50.

[33] Vetter RS, Visscher PK, Camazine S. Mass envenomations by honey bees and wasps. West J Med 1999; 170: 223-7.

[34] Triggiani M, Patella V, Staiano RI, Granata F, Marone G. Allergy and the cardiovascular system. Clin Exp Immunol 2008; 153: 7-11.

[35] Genovese A, Rossi FW, Spadaro G, Galdiero MR, Marone G. Human cardiac mast cells in anaphylaxis. Chem Immunol Allergy 2010; 95: 98-109.

[36] Benson MD, Oi H. A mild case of amniotic fluid embolism? J Matern Fetal Neonatal Med 2007; 20: 261-2.

(C) Tsunemi et al.; Licensee Bentham Open.

This is an open access article licensed under the terms of the Creative Commons Attribution Non-Commercial License (http://creativecommons.org/ licenses/by-nc/3.0/) which permits unrestricted, non-commercial use, distribution and reproduction in any medium, provided the work is properly cited. 